\title{
Propuesta de estrategia curricular para el mejoramiento de la formación metodológica del Ecuador del nivel inicial*
}

Proposal of curricular strategy for the improvement of the methodical training of the initial level Ecuador



Rogelio Bermúdez Sarguera ${ }^{* *}$

Lidia Patricia Estrella Acencio***

Alexandra Huerta Cruz ${ }^{* * * *}$

Rita Garcés Silva ${ }^{* * * * *}$

\section{Resumen}

El presente artículo aborda una de las problemáticas más relevantes en la formación del futuro profesional de la educación $y$, específicamente, de la educación inicial: su preparación

\footnotetext{
Artículo original derivado del proyecto de investigación titulado "Estrategias curriculares". Entidad financiadora: Universidad de Guayaquil, fecha de realización entre 2019.

** Ph.D en Educación. Universidad de Guayaquil. E-mail: rogelio.bermudezs@ug.edu.ec. Guayaquil, Ecuador. ORCID: http://orcid.org/00000003-3293-9242.

*** MSc. En Educación Universidad de Guayaquil. Guayaquil, Ecuador. E-mail: lidia.estrellaa@ug.edu.ec. ORCID: http://orcid.org/0000-0003-3628-0481.

${ }^{* * * *}$ MSc. En Educación Universidad de Guayaquil. Guayaquil, Ecuador. E-mail: alexandra.huertac@ug.edu.ec. ORCID: http://orcid.org/0000-0003-3628-0481.

${ }_{* * * * *}$ MSc. En Educación Universidad de Guayaquil. Guayaquil, Ecuador. E-mail: rita.garcess@ug.edu.ec. ORCID: http://orcid.org/0000-0002-2644-4158.
}

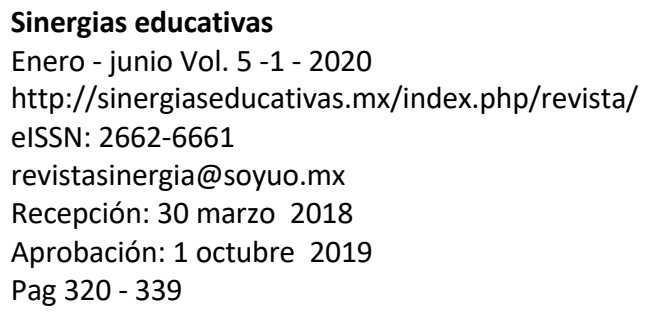

Disponible en

http://www.redalyc.org/articulo.oa?id=57356143200 24

Atribución/Reconocimiento-NoComercialCompartirlgual 4.0 Licencia Pública Internacional CC BY-NC-SA 4.0 
metodológico-investigativa. Este artículo apunta a la valoración de una propuesta metodológica con arreglo a la cual debería estructurarse la malla curricular para la formación del profesional de la educación inicial. Consideramos aplicar el método analíticosintético y el enfoque sistémico-estructural, en aras de identificar las habilidades profesionales a formar en el primer y segundo semestre, de acuerdo con las exigencias instrumentales de la carrera y la forma de evaluación que deberían adoptarse para culminar cada semestre, así como el proyecto general de investigación que ha de tributar al trabajo de titulación. La implementación de la mencionada propuesta puede pulsar el mejoramiento de la actividad metodológicoinvestigativa del estudiante de la carrera de educación inicial en su proyección científica hacia la resolución de problemas profesionales futuros.

Palabras clave: educación inicial, formación profesional, habilidades profesionales, malla curricular, perfil de egreso, propuesta metodológica.

\begin{abstract}
This article addresses one of the most relevant problems in the training of the professional future of education and, specifically, of initial education: its methodological-research preparation. This article aims at the assessment of a methodological proposal under which the curriculum mesh should be structured for the training of the initial education professional. We consider applying the analytical-synthetic method, in order to identify the professional skills to be formed in the first and second semesters, in accordance with the instrumental requirements of the race and the form of evaluation that should be adopted to complete every semester, as well as the general research project to be taxed on the work of the degree. The implementation of the aforementioned proposal may lead to the improvement of the methodological-research activity of the student of the initial education career in his scientific projection towards the resolution of future professional problems.
\end{abstract}

Key words: education, vocational training, professional skills, curriculum mesh, income profile, methodological proposal 


\section{Introducción}

En artículos anteriores, nos habíamos detenido, en primer lugar, en la caracterización de la edad psicológica de la juventud, so pretexto de demostrar que el estudiante de nuevo ingreso a la universidad no estaba facultado para responder con efectividad a los niveles de dificultad que la carrera para él establecía y, en segundo lugar, focalizamos la descripción del lugar correspondiente de la asignatura metodología de la investigación dentro de la malla curricular, en función de las habilidades profesionales previstas en el perfil de egreso de la carrera de educación inicial.

Una vez que fueron abordadas las premisas que como trabajos a este antecedieron, nos parece oportuno describir, grosso modo, las etapas que deben configurar el ejercicio investigativo de toda la carrera de educación inicial, adscrita a la facultad de Filosofía, Letras y Ciencias de la Educación, de la Universidad de Guayaquil. Dichas etapas han de conformar la propuesta de mejoramiento de todo el proceso de investigación del estudiante, a favor de su formación metodológica como futuro educador de la educación inicial.

En este sentido, nos hemos apoyado necesariamente en los aportes que en materia de aprendizaje hayan realizado la psicología, la didáctica y la pedagogía, en lo que, a leyes del aprendizaje, principio didáctico de la gradualidad formativa y dirección del aprendizaje conciernen, respectivamente. La observancia escrupulosa de los dictados científicos de cada uno de estos campos del saber, condiciona no solo la dirección del aprendizaje, sino también el decursar consecuente de este proceso por parte de quien lo lleva a cabo: el estudiante. Es poco probable defender la idea con arreglo a la cual el proceso de enseñanza podría quedar al margen del aprendizaje. Aun cuando son procesos metodológicos distintos, el caso es que inexorablemente constituyen los dos polos de una misma unidad. Y viceversa. Aunque sean los polos irrestrictos de una misma unidad, la estructura y dinámica de ambos se alejan inconmensurablemente. Por ende, de lo que se trata es de enfatizar que a estos procesos les son inherentes conocimientos diferentes y competencias distintas, con independencia de la plataforma ética y moral subyacente. 


\section{Materiales y métodos}

Si bien en todos está acuñada la idea de la unidad de los procesos de enseñanza y de aprendizaje, razón por la que se formulan en términos de una relación inseparablemente intrínseca y biunívoca, lo cierto es que estos dos procesos no son una y la misma cosa. Lo uno y lo otro se presuponen, es verdad, pero también lo uno y lo otro tienen vida propia, se excluyen. $\mathrm{Y}$ eso es crucial entenderlo para conocer los pilares sobre los que descansa nuestro proceder científicometodológico y, desde allí, armar los andamios de nuestra propuesta de mejoramiento metodológico con relación a la gestión formativainvestigativa del estudiante de la carrera de educación inicial y proceder en consecuencia.

De buenas intenciones se haya pletórico el camino hacia el infierno, reza el dicho popular. Significa que aun cuando el claustro docente de cualquier espacio universitario, o de otro nivel de enseñanza, pretenda hacer correctamente lo que su agenda profesional dicta, no necesariamente su experticia competitiva, su desempeño técnico, responde al contexto en el que ejerce su profesión, entiéndase las situaciones singulares de educación, así como las condiciones concretas bajo las que la relación docente-estudiante tiene lugar. Son más las variables intervinientes en el contexto educativo que las que pueden someterse al control del docente, como investigador.

Ahora bien, con independencia de tales premisas, el conocimiento docente-metodológico y metodológico-investigativo de un profesor deviene conditio sine qua non de la calidad de su ejercicio. Una de las leyes del pensamiento correcto, formulada por el archiconocido maestro de la antigüedad, Aristóteles, pugnaba por señalarnos que toda idea debe ser argumentada. Dicha ley, por ende, se conoce como ley de la argumentación o ley de la razón suficiente. Dicho esto, ha de presuponerse que lo que hacemos los docentes en el espacio áulico debe estar sujeto a los preceptos de argumentos filosóficos, pedagógicos, didácticos, psicológicos y lógicos, por solo mencionar algunos de los campos que indiscutiblemente se hayan imbricados con el quehacer magisterial. Eso no es para nadie un secreto. A nuestro juicio, no existe otra profesión que se sostenga en tantas y 
disímiles leyes en su dinámica hacendosa, como la profesión del educador, sea cual fuere el nivel de enseñanza en el que labore.

Para el caso de la Universidad, uno de los procesos sustantivos de mayor envergadura que allí tiene lugar, es la investigación. Si bien los procesos adyacentes a la investigación, en el contexto universitario, lo constituyen la docencia y la vinculación con la sociedad, no es difícil inferir que sin la investigación no es posible proceso de enseñanza alguno y mucho menos mejorar la comunidad, pues toda transformación de un estado actual a un estado deseado del objeto de estudio-- depende de lo que hayamos descubierto como mejora para esa singularidad. Eso es un hecho. De ahí que, sin lugar a dudas, la investigación se erija en el pivote incuestionable alrededor del cual gire toda la dinámica universitaria. $\mathrm{Y}$ eso nos aboca ineluctablemente a tener todo el conocimiento científico posible sobre lo que hizo y lo que hace que la humanidad se encuentre en este lugar, aunque haya quienes tomen sus descubrimientos para destruirla y desparecer esta especie de la faz de la tierra.

Dicho de otra manera, la universidad y la investigación no escapan de su fusión precoz, tanto en el sentido científico-metodológico como en el científico-investigativo. Como puede apreciarse, volvemos a la misma idea una y otra vez, con el firme propósito de advertir el grado de implicación y de compromiso social que a ello le es inherente.

Esa es la razón primera por la que el proceso de investigación hacia el que los estudiantes de la carrera de educación inicial se hayan implicados tiene que ser una y otra vez valorado, a favor de su formación profesional. Lamentablemente, la dinámica de este proceso transcurría, para los dos primeros semestres, con la misma intensidad y sobre los mismos parámetros impuestos al trabajo de titulación universitaria. ¿Qué pretendemos decir con esto?

En primer lugar, que el proceso de investigación llevado a cabo en los dos primeros semestres de la mencionada carrera, le asistían los mismos requerimientos que la investigación proyectada para culminar los estudios universitarios. En efecto, la culminación de estudios de los dos primeros semestres, como de los semestres que 
siguen de inmediato, sería evaluada a raíz de la presentación y defensa, ante el claustro profesoral de la carrera, de un informe de investigación cuya estructura respondía literalmente a los cánones previstos para el trabajo de titulación, previsto a las alturas del noveno semestre. Así, vulnerando cualquier vestigio de sobriedad y mesura metodológica, a juicio nuestro, el estudiante resultaba prisionero de exigencias que no le pertenecían. Dicho más claramente, ¿cómo pedirle a un estudiante del primer semestre que ejecute ejercicios, y más en el plano metodológico-investigativo, que únicamente le compete al del noveno semestre? ¿Dónde quedó la historia del continuo saber del alumno? ¿Es que no se hace necesario el transcurso del estudiante por todos y cada uno de los semestres del quehacer universitario, de los que configuran una carrera, para llegar a ejecutar una investigación del alcance de la del noveno semestre? ¿Cómo ingresar a la Universidad, si aún no hemos aprobado los saberes de los grados inferiores? ¿Es que, en el estudiante, quizás considerado tábula rasa de los espacios áulicos, puede ser responsable ante aquello para lo cual aún no está preparado y en el que se pueden escribir jeroglíficos debido a su no sensibilidad ante lo que se enseña? ¿Por qué pedir a los estudiantes de nuevo ingreso que resuelvan problemas que ni los de salida pueden resolver con toda certeza y responsabilidad? ¿Qué principios científicometodológicos se han violado en la enseñanza y el aprendizaje, redundado en el comportamiento inseguro estudiantil al que asistimos por la "injusticia" curricular? Muchos más cuestionamientos de rigor no se harían esperar, pero una idea sí lograría sintetizar lo que sin ambages pretendemos decir: no le exijamos al joven del primer semestre lo que instituido está para el de noveno.

Ello está dado, ante todo, por las propias características de la juventud como edad psicológica, como tiene que ver con los procedimientos metodológicos aplicados en el contexto de enseñanza $\mathrm{y}$, en consecuencia, por los procedimientos metodológicos que el estudiante debe ejecutar al aprender.

Sobre la caracterización psicológica del estudiante de nuevo ingreso a la universidad, hubimos de detenernos en un artículo anterior, 
redactado aparte, debido a lo exiguo de la extensión con que contamos para exponer nuestras ideas sobre este objeto de análisis.

En lo que respecta a los procedimientos metodológicos de la enseñanza y los que se promueven en el aprendizaje debido a ella, es probable que no hayan sido advertidos determinados principios que incuestionablemente impone tanto una gestión como la otra. Si concebimos la enseñanza como la dirección del aprendizaje y el aprendizaje como la modificación de los recursos cognitivos e instrumentales del estudiante (Bermúdez y Rodríguez, 2009a), entonces bien puede observarse que la figura del docente deja de ser protagónica en el aula para devenir la persona que facilita dicha modificación. Y cuando nos referimos al término facilitación, estamos aduciendo a que lo que sucede en el estudiante al aprender, no es una ecuación matemática que deviene función de lo que hace el docente. Si así fuese, resulta pueril la idea de que la plenitud de los estudiantes alcanzaría el más alto rendimiento académico. Y nada más lejos de la verdad.

El proceso de aprendizaje, querámoslo o no, debe hallarse bajo la égida de, al menos, tres principios fundamentales, a saber,

a) La ley psicológica del ejercicio,

b) El principio didáctico de la asequibilidad de los contenidos y

c) El principio didáctico de la derivación gradual de los objetivos de aprendizaje.

Pudiésemos abordar otras leyes y principios también relevantes, en el campo de la pedagogía, la psicología y la didáctica, pero de lo que se trata es de focalizar aquellos preceptos sin los que el ejercicio del aprendizaje no puede tener lugar. Detengámonos brevemente en cada uno de ellos.

\section{Resultados}

a) Ley psicológica primaria del aprendizaje: ley del ejercicio 
De acuerdo con esta ley, formulada en la palestra psicológica por el investigador norteamericano E. Thorndike, las probabilidades que de que una respuesta suceda a una situación dada -a un estímulo determinado--, dependen del número de contactos -conexiones-- que se establece entre esa respuesta y el estímulo (Bermúdez y Rodríguez, 2005). En términos pedagógicos, la ley está aludiendo a que para llegar a aprender algo, es necesario que la situación de aprendizaje se le presente al alumno el número de veces que prescribe ese aprendizaje. También conceptualizada como ley de la repetición, de la práctica o ley del uso y el desuso, lo cierto es que para aprender algo se necesita que la situación se repita tantas veces como la persona -o el animal- lo necesite. Esto trae a colación, igualmente, que "...no se puede obviar el carácter individualizado de las repeticiones [la cursiva es añadida]-dependiendo del semestre de los estudiantes--, como tampoco puede soslayarse que las repeticiones dependen del tipo de aprendizaje [la cursiva es añadida] que en la persona tendrá lugar” (Bermúdez y Rodríguez, 2005, p.35). En resumen, "...si para aprender son necesarias las repeticiones y cada cual aprende a su propio ritmo, entonces las repeticiones serán una función de los recursos personales [la cursiva es añadida] con los que el individuo cuenta" (Bermúdez y Rodríguez, 2005, p.35).

De aceptar lícita esta idea en el contexto educacional, ¿cómo pretender entonces que un estudiante del primer semestre de una carrera universitaria realice lo que prescrito está para uno del noveno? ¿Con qué recursos cognitivos e instrumentales cuenta el estudiante del primer año universitario para enfrentar problemas con perfiles de noveno? En otras palabras, ¿con cuáles conocimientos y habilidades cuenta el alumno para resolver las tareas que se les formulan en la palestra universitaria de un primer y segundo semestres? En última instancia, ¿cómo planificar, ejecutar y redactar el informe de investigación de un proyecto de integración de saberes para un estudiante que acaba de ingresar en la casa de altos estudios?, ¿De qué conocimientos y habilidades metodológicas investigativas podrían hablarse, si el estudiante no ha ejecutado trabajo colaborativo o autónomo alguno en los que el objeto de estudio haya sido el aprendizaje del diseño teórico y metodológico de una investigación con su ejecución y redacción del informe correspondiente? $\mathrm{Y}$ aquí no estamos focalizando con toda 
intencionalidad el hecho de que sin la repetición de las acciones investigativas no se producen las habilidades profesionales para la investigación. Querámoslo o no, esta es una verdad de Perogrullo. "La madre de la enseñanza, --afirmaba el conocido filósofo griego Aristóteles- es la repetición".

b) El principio didáctico de la asequibilidad de los contenidos

A nuestro juicio, este principio didáctico se halla estrechamente imbricado con la ley de la pertinencia de la psicología del aprendizaje. La ley dicta, en su postulado basal, que "las conexiones (E-R) que se pertenecen en forma natural son mejor aprendidas que las que no se pertenecen por sus elementos" (Bermúdez y Rodríguez, 2005, p.36). Desde la didáctica, bien se avizora que no se puede aprender un conocimiento o una habilidad, si antes no se han producido los que obligatoriamente deben haberse sucedido. No es difícil advertir también el tan cacareado principio de los conocimientos previos o el aprendizaje significativo que el autor norteamericano D.Ausubel hubo de considerar en la contemporaneidad. En su fundamentación metodológica, por cierto, nada novedosa, pues sobre ello ya había irrumpido el también psicólogo norteamericano E. Thorndike, en 1896, acerca de la relación del nuevo conocimiento con el ya aprendido, este investigador afirmaba:

...el aprendizaje de nuevos conocimientos se basa en lo que ya se conoce [la cursiva es añadida]. Es decir, la construcción del conocimiento comienza con nuestra observación y reconocimiento de eventos y objetos a través de conceptos que ya tenemos [la cursiva es añadida]. Aprendemos construyendo una red de conceptos y agregándolos [la cursiva es añadida]. (Ausubel, Citado en Universitas Pendidikan Indonesia, 2019, p.1)

De aquellos polvos vienen estos lodos, reza el aforismo popular. Y en efecto, a todo le es inherente una historia, etapas -momentos, fases-- a través de las cuales se suceden los hechos. ¿Cómo podríamos aprender a multiplicar, si no sabemos sumar ni restar?, ¿cómo resolver integrales matemáticas, si no hemos aprendido a resolver diferenciales del mismo orden?, ¿cómo escribir un informe de investigación, si aún no ha aprendido a redactar textos de obvia 
cientificidad?, ¿cómo ascender a niveles de órdenes superiores, si no hemos transitado por los niveles de órdenes inferiores que a aquellos sostienen?, ¿cuándo lo superior surgió sin la presencia de lo inferior, que, además, dentro de aquel primero late de manera sempiterna?, ¿cómo borrar la historia del desarrollo tecnológico actual? Por lo tanto, no existe sortilegio misterioso alguno que defienda la idea de que podamos aprender cualquier conocimiento o habilidad por arte de magia. Estos malabarismos metodológicos no constituyen recursos de aprendizaje de un estudiante y, mucho menos, del estudiante de la educación superior. Dicho brevemente, para llegar al noveno semestre, hay que pasar por el primero y el segundo, de lo que se infiere que los aprendizajes de estos dos primeros deben sostener los del noveno, deben ser justamente los primeros escalones en la ascensión. $\mathrm{O}$ dicho a la inversa, los aprendizajes del noveno semestre de una carrera universitaria se sostendrán infaliblemente sobre la base de los que ya ocurrieron con anterioridad. En síntesis, un conocimiento o una habilidad no resultan asequibles, alcanzables, para quienes no han aprendido primero la base teórica y/o metodológica que a ellos obligatoriamente subyacen. Por lo tanto, ¿Cómo realizar un proyecto de integración de saberes, tal cual se venía realizando por el estudiante de los primeros semestres, si esos conocimientos y habilidades investigativas aún no son patrimonio de él?, ¿cómo acceder a lo que aún no ha aprendido?

c) El principio didáctico de la derivación gradual de los objetivos de aprendizaje

Todo el Universo se mueve en el sentido de la gradualidad. Solo las revoluciones, sean del tipo que fueren, identifican los saltos de una etapa a otra, generalmente agresivos, violentos, en la que prevalece la destrucción, el cataclismo caótico de lo viejo y el surgimiento de lo nuevo. Hechos así también tienen lugar en el pensamiento humano. Vale señalar que cuando se expone una nueva posición teórica que niega, en el sentido dialéctico, lo existente, generalmente se escucha decir que no se entiende de lo que se habla o que el investigador no está en sus cabales. Aun cuando se crea en la tan cacareada frase de que hay hombres que se han adelantado a su época, por las innovaciones y creatividad demostradas, lo cierto es que ellos sí han transitado por todas y cada una de las etapas de 
aprendizaje -investigación - de un objeto de estudio dado. Ah, lo que sucede es que ese investigador ha logrado ascender -en términos de abstracción-- con la rapidez propia de los hombres de ciencia. Esos hombres no se detienen en las expresiones fenoménicas de lo que se estudia, sino en su esencia, negando y abstrayéndose de lo que no es imprescindible en la dinámica del objeto de investigación. Por ende, el tiempo corre a su favor.

Para el caso de los estudiantes de nuevo ingreso a la Universidad, la situación es muy distinta. Sucede que todo debe ser enseñado en su momento y bajo la dosificación que el aprendizaje exige. Aun cuando se trate de una carrera universitaria, no se puede correr o, de lo contrario, el estudiante dejará miles de "lagunas" que no serán colmadas nunca más. No debemos olvidar a esta altura el concepto izado por la connotada investigadora italiana María Montessori, al identificar, en el desarrollo ontogénico del individuo, los llamados periodos sensitivos en el aprendizaje de determinados conocimientos. Por lo tanto, lo que debe ser aprendido ahora, no se debe dejar para después. Y lo aprendido ahora, es la base de los aprendizajes posteriores. Por ende, un aprendizaje necesariamente deriva de otro que le antecede y ello se haya sometido a la gradualidad.

Para la didáctica, esto resulta bien claro. De ahí la formulación del principio de derivación gradual de los objetivos de aprendizaje, por lo que debe entenderse la sucesión progresiva, escalonada, de los aprendizajes, tanto cognitivos como instrumentales. Cuando el estudiante formula una pregunta, ante la explicación de un concepto, digamos, no está más que exigiéndole a Ud. como docente que no se apure en la explicación, que no deje en medio del recorrido intelectual determinados elementos que le son muy necesarios para entender de qué se trata. Y está Ud. Ahora obligado a volver atrás. $\mathrm{Si}$ a Ud. le queda algo sin comprender al aprender, todo lo que sigue se vuelve oscuro e inaccesible, y mucho más si se trata de conceptos teóricos. Y ese hecho es el resultado de la violación del principio que estamos abordando. Por ejemplo, en el sentido instrumental, Ud. no puede aprender a demostrar nada, si antes no ha aprendido a valorar. Porque justo el criterio de valoración deviene posteriormente habilidad de demostración (Bermúdez y Rodríguez, en edición, 
p.53), Dicho esto, de lo que se trata es de enfatizar que no podemos aprender a demostrar nada, sin antes no hemos aprendido a valorar algo.

Este principio cardinal para el ejercicio de la profesión de maestro debiera estar lo suficientemente arraigado en la conciencia pedagógica. Sin embargo, se vulnera sin compasión alguna en la palestra magisterial, dado, entre otras cosas, por el hecho de su desconocimiento. La estructura incuestionablemente lógica coherente y consecuente - del conocimiento no da margen a saltos bruscos de esta índole, durante su aprendizaje.

No se hace difícil advertir cómo en los Programas de disciplinas y de asignaturas este principio debe estar consignado sin titubeos. Pero la mayoría de las veces, debido a la base teórica y metodológica que los sostiene, dichos programas son víctimas de falencias significativas en aquel sentido y dan al traste con lo que se exige en la preparación del futuro profesional. Y, sin ambages, sabemos que esas falencias se imbrican primeramente con la problemática de la derivación gradual de los objetivos de aprendizaje, por el que se ha de entender que, dentro del sistema de orientación de dichos programas para la carrera, como lo constituye su perfil de egreso, no se hayan estructurados con plena lógica de ejecución los objetivos a conseguir. Y esa lógica descansa primordialmente en la derivación gradual como principio didáctico.

El investigador norteamericano B.F.Skinner, en los albores del siglo pasado, ya había formulado unos de los principios de extrema notabilidad en el ejercicio del aprendizaje, a saber, el principio de los pequeños pasos o aprendizaje a ritmo lento. Abriendo las puertas de su sistema teórico, este autor nos legó la excelsa idea según la cual el aprendizaje se sucede por pasos, por pequeños pasos $\mathrm{y}$, sin equivoco alguno, esos pasos se constituyen en los objetivos de aprendizaje. En sus posiciones teóricas, todo es impoluto y su expresión cimera se haya en cualquier aprendizaje necesita que se realice lentamente, paso a paso, lo que también se conoce como step by step, anglicismo ampliamente empleado en la palestra docente.

A raíz de todo lo expuesto, ha de presuponerse que el perfil del egresado para la carrera de educación inicial debe ser el documento 
contentivo de todos los objetivos de aprendizaje a conseguir y que ellos debieran estar organizados bajo el principio de la gradualidad, considerando asimismo la asequibilidad de los conocimientos, su susceptibilidad de ser aprendidos y la repetición -frecuencia y periodicidad-- a la que deben ser sometidos, tanto por su naturaleza instrumental como cognitiva. Pero no es así. De ahí nuestras proposiciones metodológicas en función del mejoramiento de la gestión de enseñanza, en aras de contribuir, en consecuencia, al mejoramiento del proceso de aprendizaje.

d) Propuesta de modificación del Proyecto de Integración de Saberes (PIS) para el 1er. y 2do. semestres de la carrera de Educación Inicial (1a aproximación al proceso de investigación)

Pensamos que el Proyecto de integración de saberes (PIS), para el 1er. y 2do. semestres de la carrera de Educación Inicial, debiera quedar estructurado, en general, en tres partes constitutivas:

I. la planificación de la investigación (parte I),

II. la ejecución de la investigación (parte II) y

III. la elaboración del informe de investigación (parte III).

I. Parte: planificación

Resultados de aprendizaje:

1er. semestre

Tabla 1: "Acciones de planificación de la investigación"

\begin{tabular}{|c|c|c|}
\hline & $\begin{array}{l}\text { Algoritmo de trabajo (tareas programadas) } \\
\text { para la planificación de la investigación por el } \\
\text { estudiante }\end{array}$ & $\begin{array}{l}\text { Cronograma } \\
\text { de ejecución }\end{array}$ \\
\hline 1 & Identificación de la situación problémica. & \\
\hline 2 & $\begin{array}{l}\text { Trabajo con las fuentes bibliográficas (para la } \\
\text { búsqueda de la información) }\end{array}$ & \\
\hline
\end{tabular}






elaboración propia

II. Parte: ejecución de la investigación

Tabla 2: "Acciones que tributan a la ejecución de la investigación"

\begin{tabular}{l|l|l}
\hline 1 & $\begin{array}{l}\text { Algoritmo de trabajo (tareas programadas)para la } \\
\text { ejecución de la investigación }\end{array}$ & $\begin{array}{l}\text { Cronograma } \\
\text { de ejecución }\end{array}$ \\
\hline 2 & Formular la situación problémica identificada. & $\begin{array}{l}\text { Seleccionar la familia -escuela, comunidad- que será } \\
\text { objeto de estudio, como contexto que promueve la } \\
\text { educación inicial del niño. }\end{array}$ \\
\hline 3 & $\begin{array}{l}\text { Aplicar el método de observación participante, en aras } \\
\text { de la recopilación de la información requerida. }\end{array}$ & \\
\hline 4 & $\begin{array}{l}\text { Analizar (procesar) los datos (información) obtenidos - } \\
\text {-aplicación de métodos de procesamiento de la } \\
\text { información recopilada: a) Métodos intelectuales de } \\
\text { procesamiento de información: § análisis-síntesis, } \S \\
\text { histórico-lógico, § enfoque de sistema b) Métodos } \\
\text { estadísticos de procesamiento de información: } \S \\
\text { mediana, § moda § media aritmética }\end{array}$ \\
\hline
\end{tabular}

elaboración propia

Condiciones de aplicación del método

Observaciones:

$\S$ Como en el 1er. semestre no hay prácticas pre-profesionales (PPP), se recomienda que los estudiantes apliquen la guía de observación elaborada en clase, en sus horas de trabajo autónomo.

$\S$ Para ello, deberán seleccionar una institución de Educación Inicial, de modo que se le permita la aplicación de la guía de observación elaborada en el espacio áulico e identificar, con ella, los indicadores e ítems que tradujeron la organización académica de la institución y su infraestructura, como las únicas dos dimensiones requeridas a registrar en la observación. 
III. Parte: elaboración del informe del PIS

En aras de la presentación de los resultados de aprendizaje, el informe debe redactarse a manera de ensayo y bajo el algoritmo siguiente:

Propuesta de implementación del PIS para los semestres 1 o y 20

Tabla 3: "Acciones pertinentes a la elaboración del informe de investigación (ensayo)"

\begin{tabular}{|c|c|c|c|}
\hline & $\begin{array}{lr}\text { Estructura } & \text { del } \\
\text { informe } & \text { de } \\
\text { investigación(ensayo) }\end{array}$ & $\begin{array}{l}\text { Contenido de cada una de } \\
\text { las partes del ensayo,como } \\
\text { informe de investigación }\end{array}$ & $\begin{array}{l}\text { Cronograma } \\
\text { de ejecución }\end{array}$ \\
\hline 1 & Introducción & $\begin{array}{l}\text { Breve bosquejo histórico de } \\
\text { la problemática. Objetivos } \\
\text { (general y específicos). }\end{array}$ & \\
\hline 2 & Desarrollo: (parte I) & $\begin{array}{l}\text { Concepciones teóricas sobre } \\
\text { las políticas educativas. }\end{array}$ & \\
\hline 3 & Desarrollo: (parte II) & $\begin{array}{l}\text { Concepción metodológica } \\
\text { sobre la observación como } \\
\text { método de investigación. (La } \\
\text { guía de observación debe ser } \\
\text { un elemento sine qua non de } \\
\text { esta parte II). }\end{array}$ & \\
\hline 4 & Desarrollo: (parte III) & $\begin{array}{ll}\text { Procesamiento de la } \\
\text { información recopilada. }\end{array}$ & \\
\hline 5 & Conclusiones & $\begin{array}{l}\text { Redactar la información en } \\
\text { formadeensayo, de acuerdo } \\
\text { con los objetivos propuestos. } \\
\text { Tomar en cuenta las normas } \\
\text { de citación(aprendizaje de } \\
\text { las normas APA, edición } 6 \text { - } \\
\text { para redactar, citar y } \\
\text { estructurar la información en } \\
\text { el informe de investigación). }\end{array}$ & \\
\hline 6 & Anexos & Fotos, grabaciones, etc. & \\
\hline
\end{tabular}

Elaboración propia

Observación:

$\S$ Para el 2do. semestre, se cumplen los mismos algoritmos que para el 1ro., solo que bajo la aplicación del método historias de vida. 
$\S$ Para el 2do. semestre, debiera añadirse, a la estructura del ensayo, un acápite que reflejara las recomendaciones del estudiante al sujeto investigado, pulsados por las impresiones diagnósticas realizadas.

Por último, no sería ocioso terminar nuestra propuesta, sin antes presentar nuestras consideraciones para toda la carrera, cumpliendo las máximas como principios ya esgrimidos.

Propuesta de implementación perspectiva del PIS para toda la Carrera

Tabla 4 "Estrategia curricular semestral para el dominio de las habilidades profesionales investigativas"

\begin{tabular}{|c|c|c|c|}
\hline $\begin{array}{lr}\text { Habilidad } & \\
\text { investigativa } & \mathrm{a} \\
\text { formar } & \mathrm{y} / \mathrm{o} \\
\text { desarrollar } & \\
\end{array}$ & Semestres & Asignaturaspredominantes & Evaluación \\
\hline $\begin{array}{l}\text { Trabajo con las } \\
\text { fuentes (elaborar } \\
\text { fichas } \\
\text { bibliográficas, } \\
\text { emplear las normas } \\
\text { de citación o } \\
\text { referencias) }\end{array}$ & 1 у 2 & $\begin{array}{l}\S \text { Lectura y escritura } \\
\text { académica I } \S \text { Lectura y } \\
\text { escritura académica II }\end{array}$ & $\begin{array}{l}\text { Informes escritos } \\
\text { (ensayos), trabajos } \\
\text { referativos, } \\
\text { seminarios, etc. }\end{array}$ \\
\hline $\begin{array}{l}\text { Elaboración de un } \\
\text { diseño } \\
\text { investigación de } \\
\text { (parte I de su } \\
\text { estructura } \\
\text { general): § Diseño } \\
\text { teórico } \\
\text { (epistémico) }\end{array}$ & 3 & $\begin{array}{l}\S \quad \text { Proceso } \\
\text { investigación: } \\
\text { Planificación del } \\
\text { (diseño teórico de la } \\
\text { investigación) }\end{array}$ & $\begin{array}{l}\text { Defensa del Diseño } \\
\text { (teórico) elaborado }\end{array}$ \\
\hline $\begin{array}{l}\text { Seleccionar } \\
\text { métodos (técnicas) } \\
\text { de investigación } \\
\text { (observación e e } \\
\text { historias de vida) }\end{array}$ & 4 & $\begin{array}{l}\text { § Metodología de la } \\
\text { investigación: observación } \\
\text { con instrumentos } \$ \\
\text { Metodología } \\
\text { investigación: Historias de } \\
\text { vida § Metodología de la } \\
\text { investigación: Técnicas } \\
\text { dialógicas }\end{array}$ & $\begin{array}{l}\text { Informes escritos, } \\
\text { historia psicosocial } \\
\text { (historias de vida) }\end{array}$ \\
\hline $\begin{array}{l}\text { Elaboración de un } \\
\text { diseño de } \\
\text { investigación(parte } \\
\text { II de su estructura } \\
\text { general): § Diseño } \\
\text { metodológico }\end{array}$ & 5 & $\begin{array}{l}\S \quad \text { Proceso de } \\
\text { investigación: } \\
\text { Planificación del Diseño } \\
\text { (diseño metodológico de la } \\
\text { investigación) }\end{array}$ & $\begin{array}{l}\text { Defensa del } \\
\text { Diseño(metodológico) } \\
\text { elaborado }\end{array}$ \\
\hline
\end{tabular}




\begin{tabular}{|c|c|c|c|}
\hline $\begin{array}{l}\text { Planificacióndel } \\
\text { proyecto de } \\
\text { investigación para } \\
\text { la Titulación }\end{array}$ & 6 & $\begin{array}{l}\text { § Planificación del trabajo } \\
\text { de titulación }\end{array}$ & $\begin{array}{l}\text { Defensa del Proyecto } \\
\text { de investigación } \\
\text { elaborado }\end{array}$ \\
\hline $\begin{array}{lr}\text { Ejecucióndel } \\
\text { proyecto } \\
\text { investigación para } \\
\text { la Titulación } & \S \\
\text { Recopilación de la } \\
\text { información } \\
\text { (adecuación } \\
\text { creación } \\
\text { aplicación de } \\
\text { instrumentos los } \\
\text { investigación) } \\
\text { Procesamiento de } \\
\text { la información } \\
\text { recopilada } \\
\text { Interpretación de la } \\
\text { información } \\
\text { obtenida }\end{array}$ & 7,8 & $\begin{array}{l}\S \text { Taller de elaboración de } \\
\text { trabajos de titulación } \S \\
\text { Proceso de investigación: } \\
\text { Diagnóstico } \S \text { Proceso de } \\
\text { investigación: } \\
\text { Interpretación } \\
\text { resultados }\end{array}$ & $\begin{array}{l}\text { Artículos científicos } \\
\text { (elaboración de tablas } \\
\text { analíticas /sintéticas) }\end{array}$ \\
\hline $\begin{array}{l}\text { Redacción del } \\
\text { informe con vistas } \\
\text { a la Titulación }\end{array}$ & 9 & $\begin{array}{l}\S \text { Desarrollo del trabajo de } \\
\text { Titulación }\end{array}$ & $\begin{array}{l}\text { Informe final de } \\
\text { investigación }\end{array}$ \\
\hline
\end{tabular}

Elaboración propia

La culminación de estudios realizados en los dos primeros semestres de la carrera de educación inicial debe identificarse con la redacción científica de un ensayo. Esta forma de presentación de los resultados investigativos, lícita para la educación superior, debe predominar en estos dos primeros semestres, observando diligentemente las normas y reglas que se exigen para ello, dentro de las que denotan, en síntesis, una introducción, desarrollo y conclusiones. Esta forma de redacción de los resultados, al término de la primera aproximación de los estudiantes de 1er. y 2 do. semestres de la carrera a los procesos investigativos, debe ser descrita -o explicada-- predominantemente por la asignatura "Aprendizaje de la comunicación humana I y II".

Ya en los umbrales de estos escritos, huelga detallar aquí algunos puntos de carácter metodológico ya abordados de alguna manera, pero que, debido a su inminente notoriedad, pensamos que con ello redimiremos las posiciones tomadas sobre la culminación de estudios de los semestres a los que hemos hecho alusión todo el tiempo. Permítanos enumerarlos en el orden que creemos pertinente, 
de acuerdo con la estrategia metodológica general de enunciado estructural propuesta.

1. Cabe observar que la presente estrategia, tal y como está concebida, requiere de la modificación de determinados programas, con los subsiguientes cambios en el Plan de estudios. Por lo tanto, no se debe ejecutar de inmediato tal cual se describe, pero es importante crear las condiciones para su futura implementación, especialmente, en lo que concierne a la preparación metodológica de los docentes de todas las asignaturas de la carrera.

2. La elaboración del PIS debiera limitarse de manera fidedigna al algoritmo propuesto, pues el estudiante no posee aún las competencias profesionales suficientes como para elaborar un trabajo científico de grandes dimensiones y de manera independiente.

3. Solo debe tomarse en cuenta la sujeción del claustro profesoralestudiantil a los dictados sobre los que descansará la ejecución y la elaboración del informe del Proyecto (ensayo), de modo que se constriñan de manera estricta a las proposiciones teóricometodológicas refrendadas en la asignatura de Metodología de la investigación. ¡Y nada más!

4. El ejercicio de formación del profesional de la educación inicial, como cualquier otro de la educación superior, debe considerar de modo riguroso los principios didácticos de lo simple a lo complejo, así como la accesibilidad y asequibilidad a los conocimientos que se abordan. De ello se infiere que no deben ser empleados métodos de investigación --ni conocimientos teóricos-- que no hayan sido ya abordados con todo el rigor en las situaciones de aprendizaje programadas para estos primeros semestres.

5. La lesson study, como metodología dirigida al trabajo grupal, de un número reducido de maestros, en aras de discutir las metas de aprendizaje y planificar una lección de clase real, prevista en el 1er. semestre de la carrera, debe ser negada de los contenidos de la materia metodología de la investigación, pues ella constituye un medio metodológico de enseñanza, no de investigación. 


\section{Conclusiones}

De acuerdo con los resultados del análisis realizado, sería sugerente y metodológicamente estimable que los estudiantes de 1ro. y 2 do. semestres no realicen Proyectos de Integración de Saberes, tal cual se ha venido ejecutando hoy día, más que el que corresponde a un primer nivel, en el que los conocimientos abordados y las competencias aprendidas condicionen la preparación metodológica del estudiante para el ejercicio pre-profesional. En su lugar, proponemos que se redacte, con la severidad requerida, un ensayo de culminación de estudios para los dos semestres iniciales de la carrera.

$\S$ La elaboración del PIS debe responder, estrictamente, a los conocimientos y habilidades que la asignatura metodología de la investigación científica establece para cada semestre. No existe razón alguna para que sobre el estudiante "cuelguen" exigencias que no son inherentes a ese periodo de aprendizaje.

$\S$ Las asignaturas restantes de la malla curricular, correspondientes al 1ro. y 2do. semestres de la carrera de educación inicial, aportan a dicho proyecto desde sus contenidos y desde la ejecución frecuente y periódica de las mismas habilidades que le son imprescindibles dominar a un educador del nivel inicial para el ejercicio de la profesión.

$\S$ La elaboración del PIS debe estructurarse por etapas, de modo que se respete rigurosamente el principio didáctico de gradualidad del aprendizaje de conocimientos y habilidades profesionales, así como el principio psicológico de los pequeños pasos y la ley del ejercicio.

$\S$ No deben desestimarse otras leyes y principios teóricos y metodológicos que han de prevalecer en los contextos de enseñanza $\mathrm{y}$ de aprendizaje, aun cuando nos hemos hechos eco principalmente de los ya expuestos. 


\section{Referencias}

Bermúdez Sarguera, R. y Rodríguez Rebustillo, M. (2019). "Psicología del conocimiento científico". ( $3^{\mathrm{a}}$ edición). Cienfuegos: Universo Sur.

Angulo de Haro, D. (2005). "Las leyes del aprendizaje". (1ª edición). La Habana: Pueblo y educación.

Universitas Pendidikan Indonesia. (2019). "Ausubel's Learning Theory”. Indonesia: Universitas Pendidikan Indonesia. Recuperado de http://fpmipa.upi.edu/data/report_activity/9875881844.pdf 